\title{
Crystal structures of the psychrophilic $\alpha$-amylase from Alteromonas haloplanctis in its native form and complexed with an inhibitor
}

\author{
NUSHIN AGHAJARI, ${ }^{1,3}$ GEORGES FELLER ${ }^{2}$ CHARLES GERDAY, $^{2}$ AND RICHARD HASER ${ }^{1,3}$ \\ ${ }^{1}$ Laboratoire d'Architecture et Fonction des Macromolécules Biologiques, UPR9039, Institut de Biologie Structurale et \\ Microbiologie, IFR1, CNRS, 31 Chemin Joseph Aiguier, 13402 Marseille cedex 20, France \\ ${ }^{2}$ Laboratoire de Biochimie, Institut de Chimie B6, Université de Liège, Sart-Tilman B-4000 Liège, Belgium
}

(Received October 8, 1997; ACCEPTED December 2, 1997)

\begin{abstract}
Alteromonas haloplanctis is a bacterium that flourishes in Antarctic sea-water and it is considered as an extreme psychrophile. We have determined the crystal structures of the $\alpha$-amylase (AHA) secreted by this bacterium, in its native state to $2.0 \AA$ resolution as well as in complex with Tris to $1.85 \AA$ resolution. The structure of AHA, which is the first experimentally determined three-dimensional structure of a psychrophilic enzyme, resembles those of other known $\alpha$-amylases of various origins with a surprisingly greatest similarity to mammalian $\alpha$-amylases. AHA contains a chloride ion which activates the hydrolytic cleavage of substrate $\alpha-1,4$-glycosidic bonds. The chloride binding site is situated $\sim 5 \AA$ from the active site which is characterized by a triad of acid residues (Asp 174, Glu 200, Asp 264). These are all involved in firm binding of the Tris moiety. A reaction mechanism for substrate hydrolysis is proposed on the basis of the Tris inhibitor binding and the chloride activation. A trio of residues (Ser 303, His 337, Glu 19) having a striking spatial resemblance with serine-protease like catalytic triads was found $\sim 22 \AA$ from the active site. We found that this triad is equally present in other chloride dependent $\alpha$-amylases, and suggest that it could be responsible for autoproteolytic events observed in solution for this cold adapted $\alpha$-amylase.
\end{abstract}

Keywords: allosteric activation; cold adaptation; crystal structure; glycosyl hydrolases; inhibition; psychrophilic

$\alpha$-Amylases $\alpha$-1,4-glucan-4-glucanohydrolase, EC 3.2.1.1 are endoglucanases widely distributed in bacteria, fungi, animals, and plants. They catalyze the hydrolysis of starch, glycogen, and related polysaccharides by cleaving internal $\alpha$-1,4-glycosidic bonds. In addition to their biochemical interest, $\alpha$-amylases have a number of important biotechnological applications in food and starch processing industries (Vihinen \& Mäntsälä, 1989).

Alteromonas haloplanctis is a gram negative bacterium collected in Antarctica and secreting a calcium- and chloride-dependent $\alpha$-amylase. It grows at sub-zero temperatures in its natural environment and can be considered as an extreme psychrophile (Feller et al., 1992). The psychrophilic $\alpha$-amylase is characterized by a high catalytic efficiency which compensates for the reduction of chemical reaction rates inherent to low temperatures. In addition, all biophysical parameters recorded suggest a flexible conformation of this heat-labile $\alpha$-amylase. The two crystal structures reported here are the first three-dimensional structures of a psy-

Reprint requests to: Richard Haser, Institut de Biologie et Chimie des Protéines, UPR412, CNRS, 7 Passage du Vercors, 69367 Lyon Cedex 07, France; e-mail: r.haser@ibcp.fr.

${ }^{3}$ Present address: Institut de Biologie et Chimie des Protéines, UPR412, CNRS, 7 Passage du Vercors, 69367 Lyon Cedex 07, France. chrophilic enzyme; moreover, they provide a number of significant features related to various aspects of biochemistry and microbiology. The structure of $A$. haloplanctis $\alpha$-amylase complexed with Tris allows to understand the molecular basis of glycosidase inhibition by this cation which is a widely used compound in biochemistry. This bacterial $\alpha$-amylase requires a chloride ion for its activity, a property previously considered as specific to mammalian $\alpha$-amylases (Brayer et al., 1995). The structure of the chlorideloaded enzyme contributes to the understanding of the effect of this allosteric activator in the catalytic mechanism. Finally, a potential serine-based catalytic triad was found at the surface, in a depression pocket of the molecule, located approximately $22 \AA$ from the amylolytic active site. Sequence alignments revealed that residues forming this triad are strictly conserved in all chloride dependent $\alpha$-amylases.

\section{Results and discussion}

\section{Molecular fold and calcium binding site}

Although isology between bacterial and vertebrate $\alpha$-amylases usually is found to be very low, $\alpha$-amylase from $A$. haloplanctis (henceforth referred to as AHA) shows significant sequence identity (50- 
$60 \%)$ with vertebrate pancreatic and salivary $\alpha$-amylases. AHA, which has the overall dimensions of $40 \AA * 50 \AA * 80 \AA$, displays the same overall fold as all $\alpha$-amylases described so far (Fig. 1). It consists of three domains; domain A being the major domain constitutes a $(\beta / \alpha)_{8}$-barrel (residues 1 to 86 and 130 to 356), domain $\mathrm{B}$ (residues 87 to 129 ) is protruding from domain A and finally the C-terminal domain (domain C) comprising residues 357 to 448 (no electron density is observed for the last 5 residues) which consists of eight $\beta$-strands. Four disulfide bridges are found in the psychrophilic $\alpha$-amylase: Cys20-Cys74, Cys120-Cys137, Cys328Cys355, and Cys402-Cys416. This is one less as compared to mammalian $\alpha$-amylases (hereafter MAA). Nevertheless, this seems to be an important difference between the bacterial (AHA) and mammalian structures (Buisson et al., 1987; Qian et al., 1993; Larson et al., 1994; Brayer et al., 1995; Ramasubbu et al., 1996), as the extra disulfide bridge in the mesophilic enzymes connects domain A (Cys 70 in MAA) to the extremity of domain B (Cys 115 in MAA) thus, limiting movements of this domain. This indeed gives the psychrophilic enzyme a larger degree of freedom in terms of overall structural flexibility. The psychrophilic enzyme binds a calcium ion, which is situated between domain A and B, in the vicinity (approximately $12 \AA$ ) of the active site. This calcium ion, which is conserved in all known $\alpha$-amylases, serves to stabilize the three-dimensional structure in this area. Binding distances for the eight-coordinated calcium ion with its ligands are summarized in Table 1. It should be noted that AHA has a $10^{4}$ times lower affinity for calcium when compared to the mesophilic counterpart from porcine pancreas (PPA) (Feller et al., 1994), although the nature of the ligands and the ligand to calcium ion distances are quite similar to those reported for MAA. One can expect the potentially higher
Table 1. Calcium and chloride binding sites

\begin{tabular}{lcc}
\hline \hline & $\begin{array}{c}\text { Wild-type AHA } \\
(\AA)\end{array}$ & $\begin{array}{c}\text { Wild-type AHA/Tris } \\
(\AA)\end{array}$ \\
\hline Distance between $\mathrm{Cl}^{-}$and & & \\
Arg 172 NE & 3.36 & 3.31 \\
Arg 172 NH2 & 3.42 & 3.43 \\
Asn 262 ND2 & 3.33 & 3.32 \\
Lys 300 NZ & 3.26 & 3.27 \\
Wat 1003 & 3.26 & 3.19 \\
Distance between Ca2+ and & & \\
Asn 88 OD1 & 2.45 & 2.41 \\
Asp 144 OD1 & 2.57 & 2.61 \\
Asp 144 OD2 & 2.56 & 2.48 \\
Gln 135 O & 2.69 & 2.63 \\
His 178 O & 2.40 & 2.54 \\
Wat 1091 & 2.45 & 2.37 \\
Wat 1107 & 2.66 & 2.65 \\
Wat 1108 & 2.52 & 2.54 \\
\end{tabular}

flexibility of this heat-labile enzyme to be the main determinant of the weak calcium coordination.

\section{Active site and catalysis}

Crystal structures of $\alpha$-amylases and related glycosyl hydrolases invariably display three essential carboxylic acids (Asp 174,

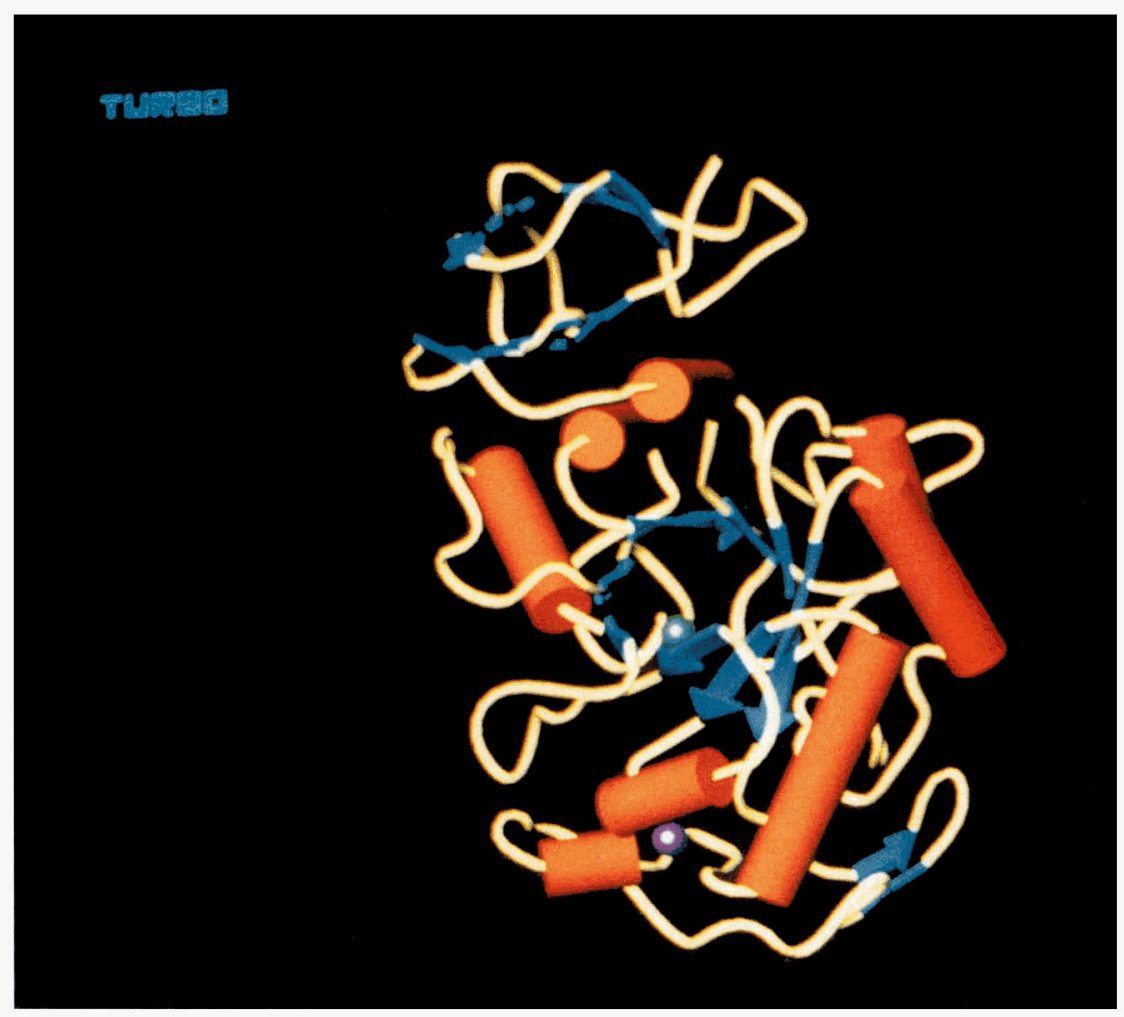

Fig. 1. The overall structure of psychrophilic A. haloplanctis $\alpha$-amylase. $\alpha$-Helices are represented as red cylinders, $\beta$-strands as blue arrows and ions as spheres: chloride in green and calcium in purple. 
Glu 200, and Asp 264, AHA numbering) in the active site (Fig. 2), which is formed as a long cleft and situated in domain $\mathrm{A}$ at the carboxyl end of the $(\beta / \alpha)_{8}$-barrel. In the currently proposed reaction mechanism, $\alpha$-amylases and closely related glycosyl hydrolases of family 13 (Davies \& Henrissat, 1995) use a pair of carboxylic acids at the active site in order to cleave the glycosidic bond with net retention of anomeric configuration via a double displacement mechanism (Koshland, 1953; McCarter \& Withers, 1996). In this reaction, the anomeric center is subjected to nucleophilic attack along with general acid catalysis and protonation of the glycosidic oxygen, resulting in the formation of a $\beta$-D-glycosyl intermediate. Hydrolysis of this intermediate by a water molecule requires general base catalytic assistance, presumably from the same residue acting as acid/base catalyst. The catalytic nucleophile has unambiguously been identified as Asp 174 (AHA numbering) in Saccharomyces cerevisiae $\alpha$-glucosidase (McCarter \& Withers, 1996), an enzyme belonging to family 13 of $\alpha$-glycosidases. There is a general agreement for the assignment of the general acid function to Glu 200 (Svensson \& Sogaard, 1993), whereas the third essential acid residue, Asp 264, possibly stabilizes the protonated state of the glutamic side chain (Strokopytov et al., 1995). When superimposing the active sites of AHA and MAA, the residues Asp 174, Glu 200, and Asp 264 in AHA and the corresponding ones in MAA (Asp 197, Glu 233, and Asp 300) superimpose perfectly with the exception of PPA. In the latter case, the side chain of Asp 300 points away from the active site in contrast to the situation in AHA, human salivary- and human-pancreatic $\alpha$-amylases, where this aspartic acid points toward the active site. Another characteristic feature of the active site is the presence of aromatic residues, which are considered as stacking partners for substrates and substrate analogues (Vyas, 1991; Kadziola, 1993; Qian et al., 1994). Aromatic residues expected to play a crucial role in substrate binding are shown in Figure 2. Tyrosine 50 in AHA, which is found at one extremity of the active site, is conserved in all $\alpha$-amylase structures available in the Protein Data Bank, thus indicating an essential role in the recognition of substrates. At the opposite end of the active site we observe a phenylalanine in AHA (Phe 223) as well as in mammalian (Buisson et al., 1987; Qian et al., 1993; Larson et al., 1994; Brayer et al., 1995; Ramasubbu et al., 1996), and plant (isozyme 2 from barley malt) (Kadziola, 1993; Kadziola et al., 1994) $\alpha$-amylases, whereas this phenylalanine has been replaced by a tyrosine in TAKA (Matsuura et al., 1984; Swift et al., 1991) and acid (Boel et al., 1990; Brady et al., 1991) $\alpha$-amylases. Finally, two consecutive tryptophans (46 and 47) are found next to Tyr 50. These are also found in MAA, whereas only one tryptophan is located here in barley $\alpha$-amylase. Interestingly, this residue is number $9(\operatorname{Trp} 9)$ in the primary structure of the latter enzyme, a feature which could never have been predicted from sequence alignments. The differences in number and nature of aromatic residues lining up the active site region could lead to specific behaviors in the catalysis resulting in different reaction products and transglycosylation patterns.

The active site of native AHA reveals a network of eight water molecules, of which four seem to be disordered, judged on the basis of a continuous electron density. A well ordered water molecule, Wat 1004, bridges Asp 264 (OD2) and Glu 200 (OE2) (Figs. 3, 4). It should be noted that this water molecule has been observed in all known three-dimensional structures of $\alpha$-amylases,

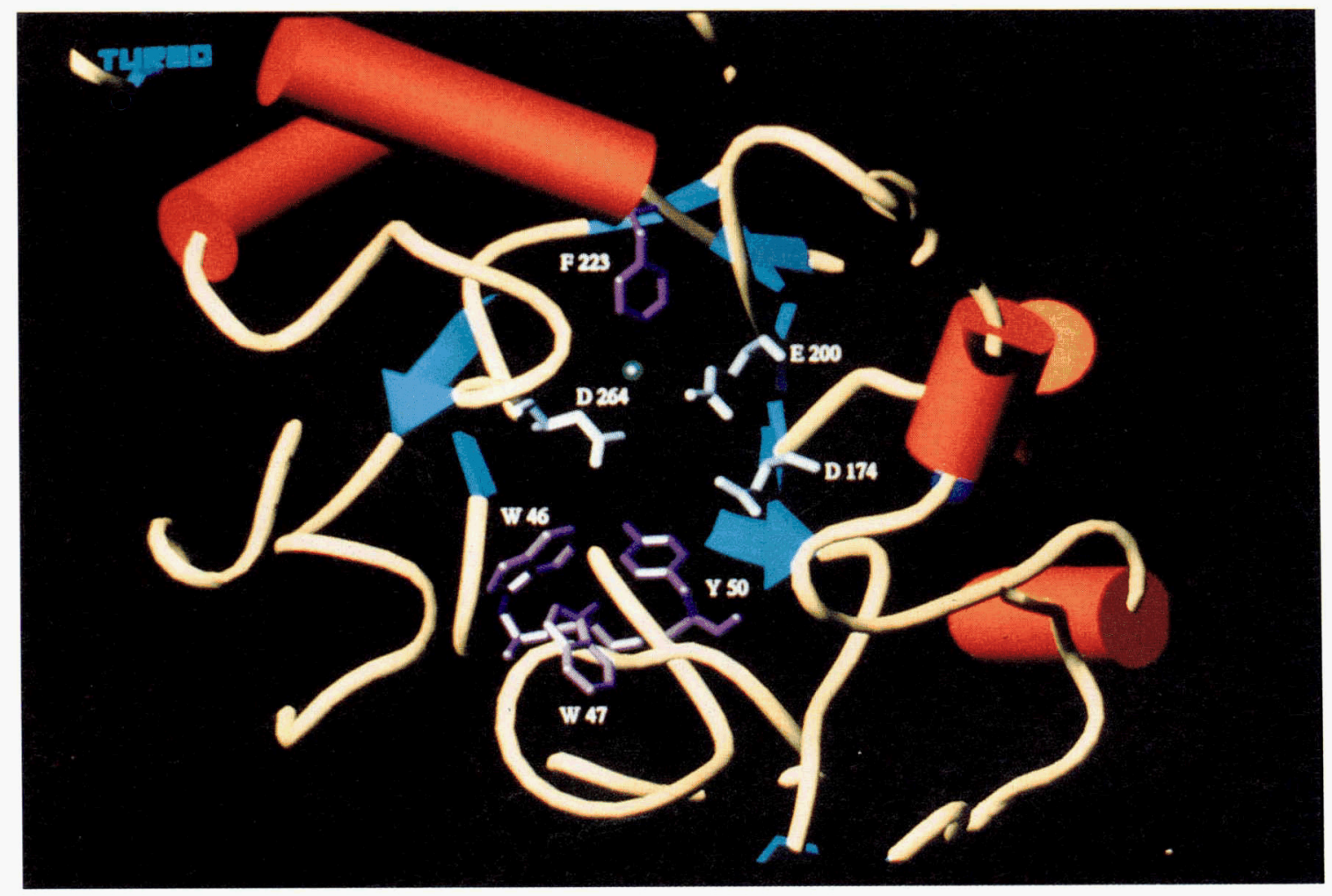

Fig. 2. The active site cleft of $A$. haloplanctis $\alpha$-amylase is situated at the carboxyl end of the $(\beta / \alpha)_{8}$ barrel. Catalytic residues are shown in white and aromatic residues expected to play an important role in substrate and/or inhibitor binding are shown in purple. 


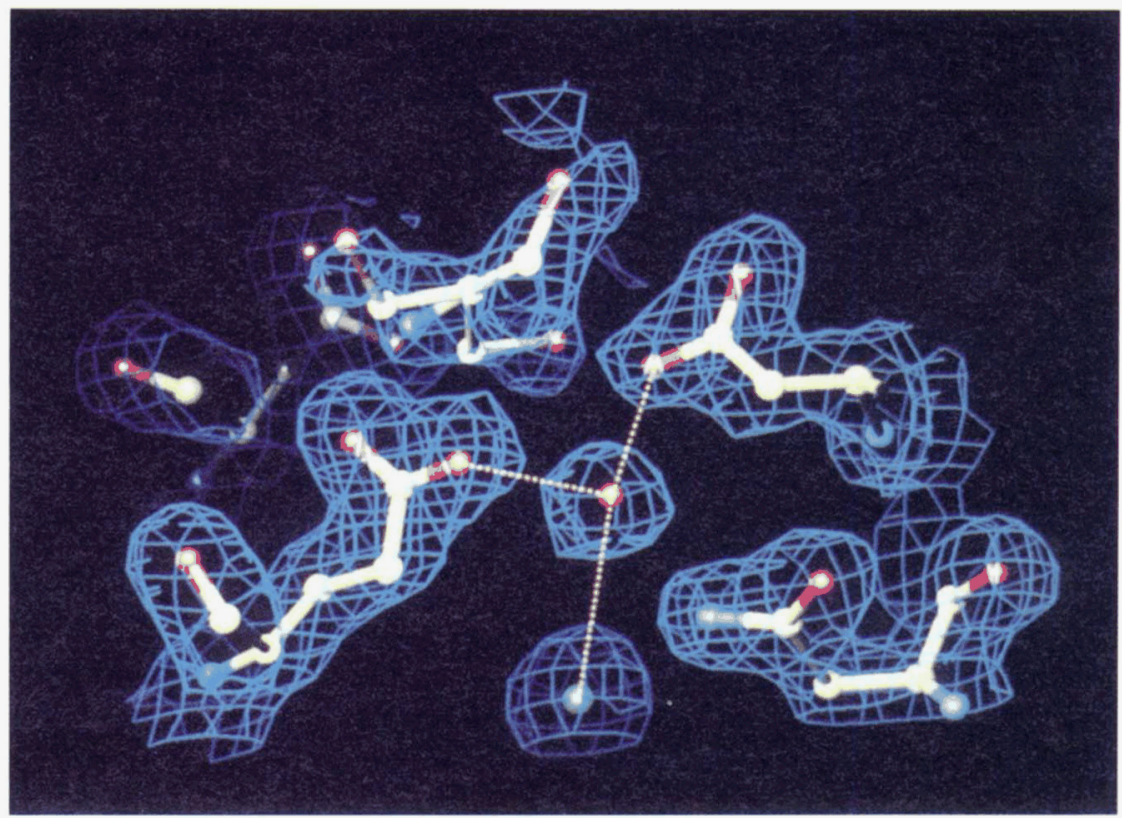

Fig. 3. Close-up on the Tris molecule bound in the active site of A. haloplanctis $\alpha$-amylase. The green sphere represents a $\mathrm{Cl}^{-}$ion. Interestingly, this $\mathrm{Cl}^{-}$ion is situated $4.6 \AA$ from a water molecule, Wat 1004 (red sphere), which is bridged between two of the catalytic residues. Glu 200 and Asp 264. This water molecule is found in all known three-dimensional structures of $\alpha$-amylases, indicating that it might play a significant role in the catalysis.

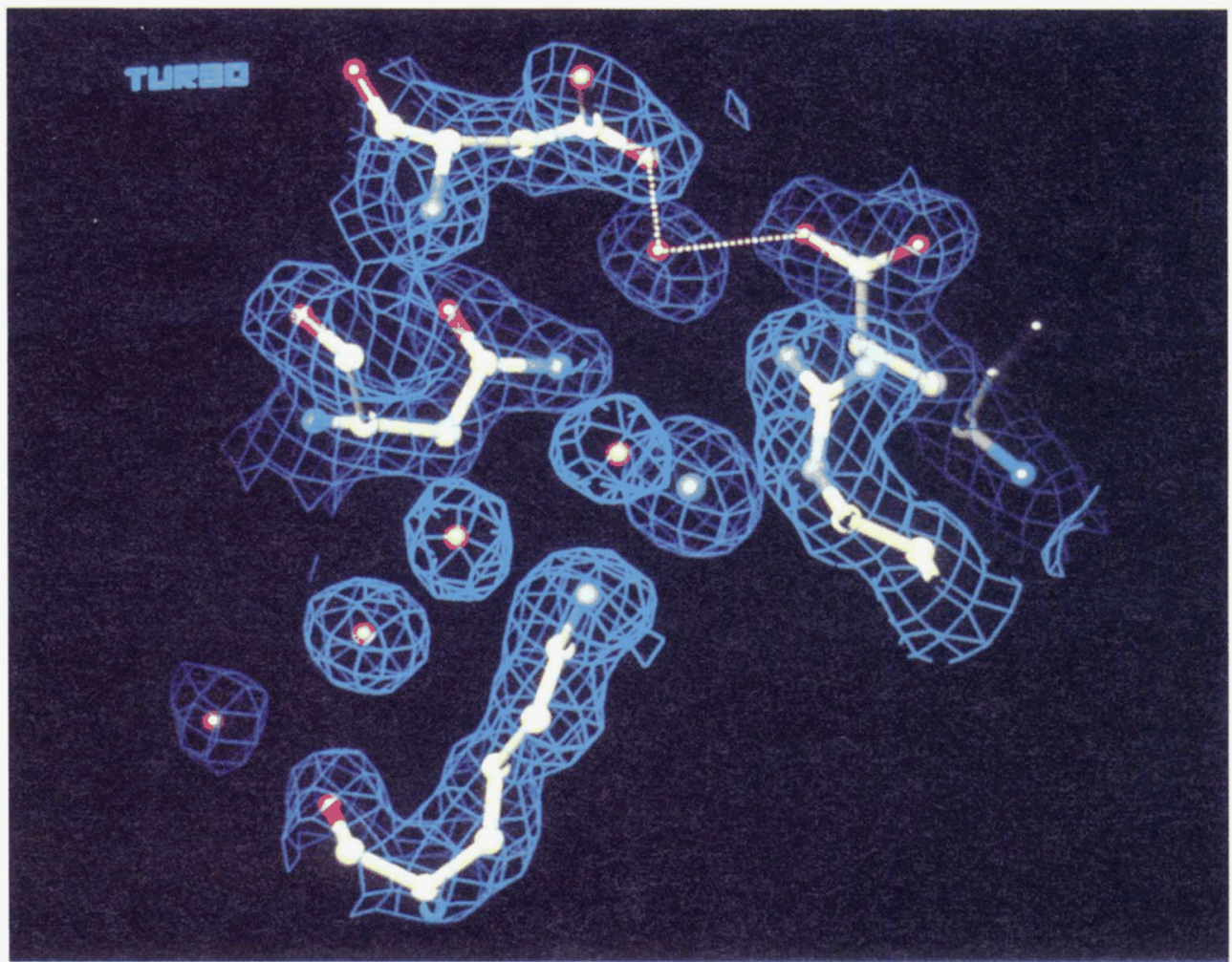

Fig. 4. The $\mathrm{Cl}^{-}$ion which is found close to the active site is shown here as a green sphere. This allosteric activator is ligated by the side chains of Arg 172 (to the right), Asn 262 (to the left), and Lys 300 (in the bottom), and a water molecule (just behind the chloride ion), Wat 1003, is found to be a fourth ligand. This water molecule is the first in a channel of six very well defined water molecules. At the top we see two of the three catalytic residues. Asp 264 and Glu 200 with Wat 1004 bridged between them. 
indicating (as suggested for the structure of barley $\alpha$-amylase (Kadziola, 1993)) that it may play a role in the catalytic process. This conserved water molecule is firmly bound to Glu 200 , which by homology to other known amylase structures is the most probable proton donor involved in the protonation step of the oxygen atom of the substrate glycosidic bond to be cleaved. After this proton transfer, the interglycosidic bond cleavage would lead to the formation of an oxocarbonium ion intermediate with a partial positive charge on the sugar carbon atom $\mathrm{C1}$, which subsequently will be covalently bound to the catalytic nucleophile, Asp 174 . Once the leaving group has departed from the active site, Glu 200 acts as a base which may be responsible for proton abstraction from Wat 1004 , inducing a hydroxyl ion ready for nucleophilic attack at the $\mathrm{C} 1$ atom. As discussed in the next section, a chloride ion situated in the near environment of this water molecule is suggested to participate in its activation, and to promote (through electrostatic repulsion) the movement of the formed hydroxyl ion toward the substrate to be hydrolyzed. It is not clear whether a chain of buried water molecules linked to the chloride ion via Wat 1003 (Fig. 4) is important for the reaction mechanism. A similar kind of water chain has been suggested to act as a water supply in the hydrolytic cleavage step in barley $\alpha$-amylase (Kadziola, 1993) and Kadziola et al. (1998).

\section{The chloride binding site and allosteric activation}

AHA requires a chloride ion for its activity. This allosteric activator is bound close to the active site and is coordinated by the side chains of Arg 172 (via NE and NH2), Asn 262 ND2, Lys $300 \mathrm{NZ}$, and a water molecule (Wat 1003) (Fig. 4; Table 1). The latter residue is an arginine in MAA, coordinating the chloride ion in a bidentate mode via NH1 and NH2 (Qian et al., 1993; Larson et al., 1994; Brayer et al., 1995; Ramasubbu et al., 1996). Site-directed mutagenesis experiments on AHA have shown that Lys 300 (Arg 337, MAA) is a key determinant of chloride binding (Feller et al., 1996a). The unidentate coordination of chloride by Lys 300 can account for the 20-fold lower binding constant reported for AHA with respect to the Arg-mediated binding to chloride in PPA. The triangular and nearly planar organization of the chloride ligands is also a striking structural feature of the anion binding site. This can indeed explain why $\mathrm{NO}_{3}{ }^{-}$and $\mathrm{SO}_{3}{ }^{-}$both having a trigonal geometry, bind even stronger than halides $\left(\mathrm{Cl}^{-}, \mathrm{Br}^{-}, \mathrm{I}^{-}\right.$, and $\mathrm{F}^{-}$) to the chloride-free enzyme. The other proteinaceous chloride ligands are conserved in almost all chloride-independent $\alpha$-amylases.

Chloride-dependent $\alpha$-amylases are almost inactive in the absence of the allosteric effector, underlining its crucial role in the catalysis. Chloride binding induces a dramatic increase of the catalytic constant $k_{\text {cat }}$ as well as a shift of the optimum $\mathrm{pH}$ of activity from $\mathrm{pH} \sim 5$ to neutrality (Thoma et al., 1971; Levitzki \& Steer, 1974; Feller et al., 1996a). The function of this allosteric effector can now be interpreted on the basis of the structural observations.

As already noted in PPA (Qian et al., 1994), chloride is bound close to Glu 233 (Glu 200 in AHA), which, as mentioned above, is the most probable proton donor in the catalytic mechanism, and may alter the $\mathrm{p} K_{a}$ value of this residue. The chloride ion (Figs. 3,4 ) is responsible for a strong electrostatic field in the vicinity of the active site residues of AHA. This anion is situated $5.4 \AA$ from Glu $200 \mathrm{CD}$ and can neutralize its partial positive charge induced by the carbonyl part of the carboxylic group. The charge effect can shift the $\mathrm{p} K_{a}$ of the group to higher values, allowing Glu 200 to remain protonated at neutral $\mathrm{pH}$ values. In addition, a network of interactions is found between chloride, the coordinating water molecule (Wat 1003), His 263 (which is thought to stabilize a transition state intermediate (Sogaard et al., 1993)) and Asp 264. The equivalent Asp 328 in CGTase has been proposed to stabilize the protonated state of the glutamic acid side chain acting as the proton donor (Strokopytov et al., 1995). We suggest that a charge relay system between the chloride ion and Asp 264 may assist the latter residue in raising the $\mathrm{p} K_{a}$ of Glu 200. Moreover, chloride coordination by Arg 172 most likely stabilizes a proton on the NH2 guanidinium group. One should note that Arg 172 is conserved not only in $\alpha$-amylases, but also in all starch hydrolases although no specific function has been assigned to this residue. In the PPAacarbose complex (Qian et al., 1994) Arg 195 NH1 (Arg 172 in AHA) is bound to OD2 of the catalytic nucleophile Asp 197 (Asp 174 in AHA). Therefore, the chloride ion, via the interaction with Arg 195, can support Asp 197 in its nucleophile function and/or in stabilizing an oxocarbonium ion intermediate. Salt bridges from Arg 172 to Asp 174 as well as to Glu 200 are present in the three-dimensional structures of native AHA and in the complex between AHA and Tris (described in the next section) as well. This indicates that the chloride ligand Arg 172 has intimate contacts with active site carboxylates and should be taken into account in future analysis of the glycosyl hydrolase reaction mechanism. Finally, it is quite remarkable that the chloride ion is in close proximity to the presumed catalytic water molecule (Wat 1004), at a distance of $4.6 \AA$. It seems more than reasonable to point out that the electrostatic field arising from this anion helps to polarize Wat 1004 as suggested above, which in turn will be deprotonated by Glu 200 . The resulting hydroxyl ion is now in a very unfavorable negative electrostatic field induced by the chloride ion and Asp 264, a situation which would favor the movement of this hydroxyl ion toward the $\mathrm{Cl}$ atom in an oxocarbonium intermediate.

Our ongoing studies on complexes of AHA with substrate analogues as well as mutants affecting the chloride binding are expected to illustrate these features in further details.

Allosteric activation by chloride has for a long time been considered as specific to mammalian $\alpha$-amylases (Brayer et al., 1995). The chloride dependence of AHA prompted us to reconsider multiple sequence alignments within the $\alpha$-amylase family. We found that the chloride ligands are exclusively conserved not only in all mammalian $\alpha$-amylases, but also in two insect $\alpha$-amylases (Anopheles gambiae and Drosophila melanogaster) and in two bacteria (A. haloplanctis and Thermomonospora curvata). Interestingly, these enzymes share extensive sequence similarity (Janecek, 1994) and obviously constitute a homogenous functional sub-family.

\section{Inhibition by Tris}

Inspection of the active site on the basis of the first collected data (see AHA/Tris Table 2) at an early stage of refinement (1.85 resolution) revealed a piece of electron density unconnected to any protein density and which could neither be assigned as noise, nor as a chain of mobile or disordered water molecules. This density allowed us to build a Tris (2-amino-2-(hydroxymethyl)-1,3 propanediol) molecule (Fig. 3). This assignment was ascertained by purification of the $\alpha$-amylase in Hepes-containing buffers, resulting in the removal of the inhibitory cation as shown by the crystal structure (crystallization is described in Materials and methods).

Superposition of the active sites of native AHA with that of the complex AHA/Tris shows that the orientations of the three catalytic residues as well as those of the other active site residues are 
Table 2. Data collection and refinement statistics

\begin{tabular}{lcc}
\hline Data set & Wild-type AHA & AHA/Tris \\
Cell dimensions $(\AA)$ & $a=71.4, b=138.7, c=115.7$ & $\mathrm{C} 222_{1}$ \\
Spacegroup & $\mathrm{C} 222_{1}$ & 153,351 \\
No. of reflections & 93,724 & 42,049 \\
No. of unique reflections & 31,485 & 1.85 \\
Resolution of data $(\AA)$ & 2.0 & $85.9 \%$ \\
Completeness (overall) & $80.5 \%$ & $79.3 \%$ \\
$l / \sigma(I)>2$ & $80.0 \%$ & $15.7 \%$ \\
$R_{\text {factor }}(\%)$ & $15.7 \%$ & $18.6 \%$ \\
$R_{\text {free }}(\%)$ & $20.4 \%$ & 0.008 \\
RMSD in bond lengths $(\AA)$ & 0.008 & 1.515 \\
RMSD in bond angles $\left({ }^{\circ} i\right)$ & 1.498 & 448 \\
No. of amino acids & 448 & 231 \\
No. of water molecules & 223 & 1 \\
No. of $\mathrm{Ca}^{2+}$ & 1 & 1 \\
No. of $\mathrm{Cl}^{-}$ & 1 & 1 \\
\end{tabular}

strictly conserved. The Tris molecule does not induce significant changes in the temperature factors of the residues to which it is bound (Fig. 5). In fact the average $B$-factor of the active site acidic residues in the native structure is $15.3 \AA^{2}$, whereas the corresponding value in the complex with Tris is $16.9 \AA^{2}$. It turns out that the hydroxyl groups, $\mathrm{OH}(1)$ and $\mathrm{OH}$ (3) of Tris, are situated at the same place as Wat 1223 and Wat 1224 , respectively, in the native structure (Fig. 5). A water molecule (Wat 1216) in the native AHA structure is found between the amino- and hydroxyl groups $\mathrm{OH}$ (2) of the Tris molecule in the structure of AHA/Tris.

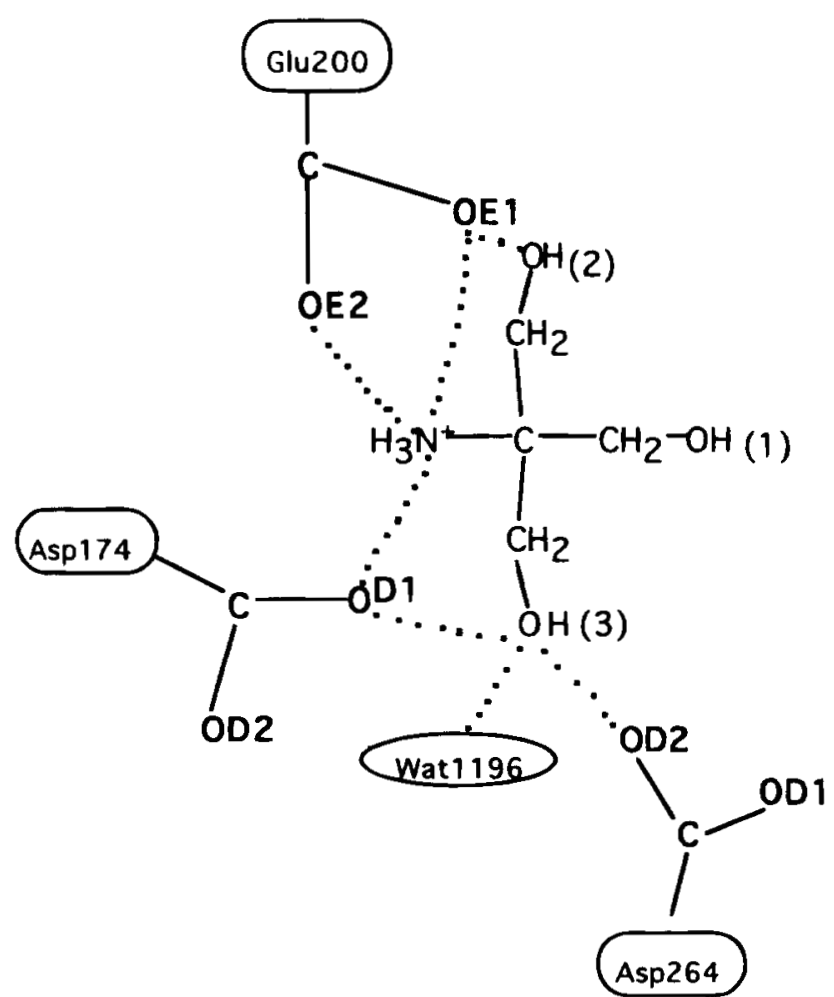

Fig. 5. Schematic representation of the residues interacting with the Tris molecule.
In fact, Tris competitively inhibits several glycosidases (Myrbäck, 1965; Jørgensen \& Jørgensen, 1967; Kersters-Hilderson et al., 1969; Chen et al., 1987) including AHA with $K_{i}=10^{-3} \mathrm{M}$ at $\mathrm{pH}$ 7.2. Inhibition studies of $\alpha$-glucosidase, trehalase, and $\beta$-fructofuranosidase have shown a 1:1 stoichiometry of the enzymeTris complex and indicated that the amino group of Tris is functioning in the inhibition and furthermore that the Tris cation rather than the free base is the true inhibitor. Most of these conclusions are confirmed by the crystal structure of the AHA-Tris complex. It is clear that only one Tris molecule is bound to the enzyme, namely in the active site where it has very strong contacts with the catalytic residues Glu 200 and Asp 174, through its amino group. Moreover, the hydroxyl groups of Tris at positions C2 and C3 are involved in hydrogen bonding interactions with the three important catalytic amino acids (Fig. 5). When the binding mode of Tris is compared to the binding mode of acarbose inhibitor (a pseudo tetra-saccharide) in complex with PPA (Qian et al., 1994), it seems clear that the above mentioned hydroxyl groups seek to adopt the same positions as the hydroxyl groups of acarbose. The amino group of Tris is situated in nearly the same environment as the nitrogen in acarbose. Keeping in mind that the nitrogen atom is responsible for the inhibitory effect in acarbose, these features most likely account for the competitive inhibition by Tris as a substrate analog. It is not possible to decide definitively whether the Tris cation or the free base is bound to AHA in the crystal structure. However, the fact that the protonated form represents about $93 \%$ at the $\mathrm{pH}$ of crystallization $\left(K_{a}=7 * 10^{-9} \mathrm{M}^{-1}\right)$ favors a bound Tris cation. Kinetic analysis of Tris inhibition in the above mentioned glycosidases also revealed that the inhibitor reacts with at least two catalytic groups having $\mathrm{p} K_{a}$ values of 3.9 and 5.8-6.8. This is obviously reminiscent of the $\mathrm{p} K_{a}$ separation of active site carboxylates in $\alpha$-amylases (Ishikawa et al., 1990).

\section{A potential proteolytic active site in AHA} and other a-amylases?

Whereas both the wild-type $\alpha$-amylase and the recombinant enzyme produced in Escherichia coli are stable for several months in Tris- $\mathrm{HCl}$ buffer containing $1 \mathrm{mM} \mathrm{CaCl}$, we observed that these enzymes rapidly are cleaved into two peptides in other buffers (Hepes, phosphate) devoid of chloride and calcium. This proteo- 
lytic cleavage is inhibited at $37^{\circ} \mathrm{C}$ by PMSF (a serine protease inhibitor), raising the possibility of an autoproteolytic activity. A systematic search for sites in the structure exhibiting a character and geometry identical to the one adopted by the catalytic residues in proteases was initiated. This search revealed a serine hydrolaselike active site (Ser 303, His 337, Glu 19), situated at the surface of the $(\beta / \alpha)_{8}$ barrel in close proximity to the C-terminal domain. As in the catalytic triad of serine proteases, the hydroxyl of Ser 303 and the carboxylate group of Glu 19 are within hydrogen bonding distance of the imidazole ring of His 337, with the major difference that the side chain of His 337 is rotated approximately $180^{\circ}$ around the $\mathrm{C}_{\alpha}-\mathrm{C}_{\beta}$ bond (Figs. 6,7 ) when compared to, for example, bovine pancreas $\beta$-trypsin. This gives a situation where His 337 ND1 is facing Ser 303 OG instead of facing the carboxylate group of Asp/Glu, and is opposite the "normal" situation in this kind of charge relay system (Blow, 1990). In a recently solved crystal structure of a subtilisin protease from Penicillium cyclopium (Koszelak et al., 1997), the side chain of the corresponding histidine was found to be rotated about $90^{\circ}$ around the $\mathrm{C}_{\alpha}-\mathrm{C}_{\beta}$ bond when compared to proteinase $\mathrm{K}$, but the authors were not certain whether this was a consequence of a PMSF molecule bound to the active serine. Considering the glutamic acid residue, the potential catalytic triad of AHA resembles the catalytic triad of lipase from Geotrichum candidum which also has a glutamic acid in the place of the usual aspartic acid (Schrag et al., 1991). Surprisingly, multiple sequence alignments also revealed that Ser, His, and Glu residues forming the triad are strictly conserved in all chloride dependent $\alpha$-amylases (i.e., all mammalian, insect, and $T$. curvata enzymes) and in Streptomyces violaceus $\alpha$-amylase. Moreover inspection of the crystal structures of human salivary (Ramasubbu et al., 1996), human pancreas (Brayer et al., 1995), and porcine pancreas (Qian et al., 1993) $\alpha$-amylases, showed a very similar triad geometry and hydrogen bond pattern involving Ser 340, His 386 , and Glu 27. Such a strict conservation of this outstanding triad is obviously not fortuitous. Further biochemical and mutagenesis experiments are in progress in order to define the function of the triad which may have profound implications regarding the key role of salivary and pancreatic $\alpha$-amylases in vertebrate physiology.

\section{Activity at low temperature}

The molecular basis of the improved catalytic activity of enzymes from psychrophilic microorganisms at low and sub-zero temperatures has received preliminary analysis based on the amino acid sequences and structural models constructed by homology modeling (Feller et al., 1994; Feller et al., 1996b; Feller et al., 1997). Only subtle structural adjustments were expected to affect either the substrate binding- and active sites or the overall molecular architecture leading to a flexible (as judged from physico-chemical data) conformation of these enzymes. The wide range of known three-dimensional structures of $\alpha$-amylases from Aspergillus oryzae (Matsuura et al., 1984; Swift et al., 1991), Aspergillus niger (Boel et al., 1990; Brady et al., 1991), porcine pancreas (Buisson et al., 1987; Qian et al., 1993; Larson et al., 1994), barley seeds (Kadziola et al., 1994), Bacillus licheniformis (Machius et al., 1995), human pancreas (Brayer et al., 1995), and human salivary (Ramasubbu et al., 1996) constitute an ideal system for studies of adaptation of enzymes to extreme conditions on a molecular level. The three-dimensional structures obtained and described in this paper provide new insights into the mechanism of cold adaptation. Indeed, superposition of AHA and PPA showed that the 24 residues

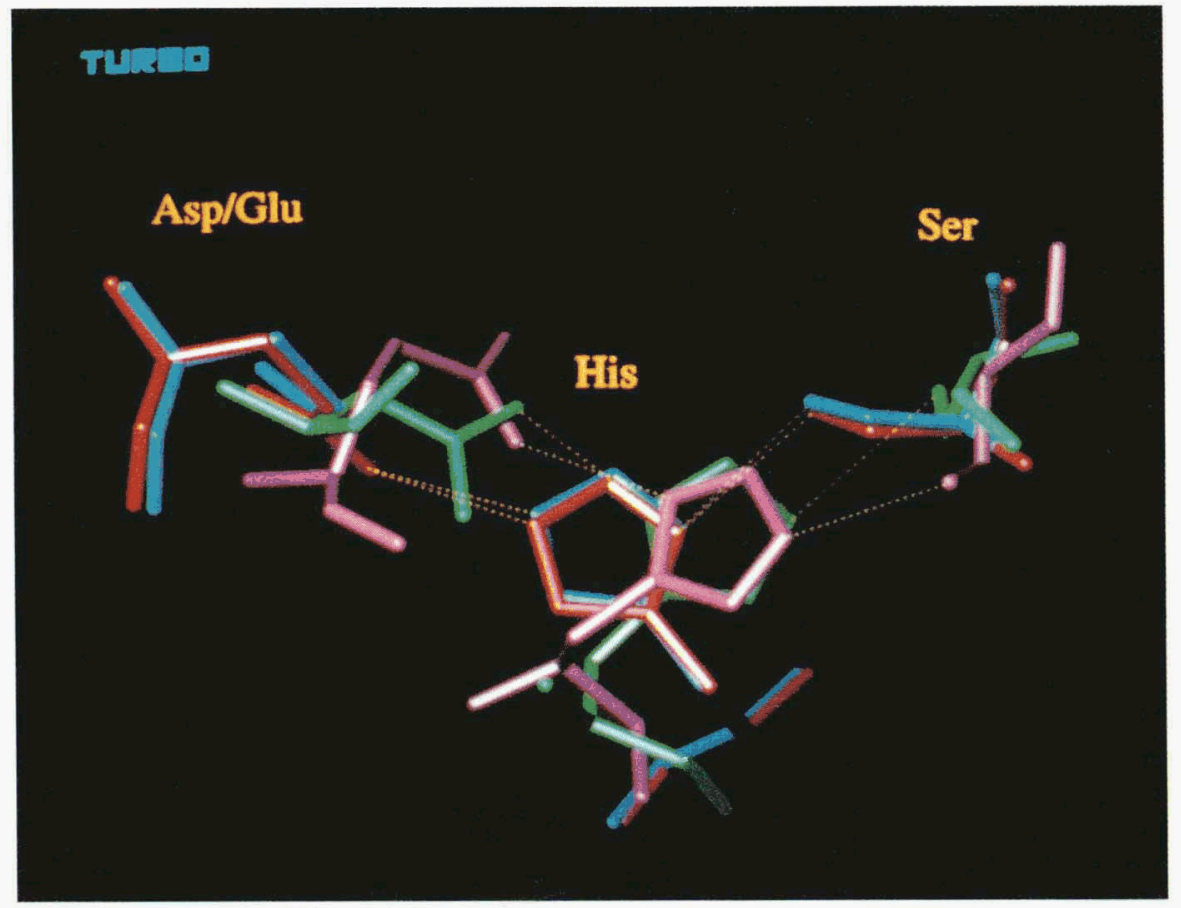

Fig. 6. The $\mathrm{C} \alpha$ backbones bearing the potential catalytic side chains of Ser 303, His 337, and Glu 19 for A. haloplanctis $\alpha$-amylase (blue) and the corresponding residues for $G$. candidum lipase (pink), human salivary $\alpha$-amylase (red), and bovine pancreas $\beta$-trypsin (green) are shown superimposed. The three-dimensional structures for GCL, HSA, and BPT were found in the Protein Data Bank under entry codes: 1THG, ISMD, and 1TLD, respectively. 


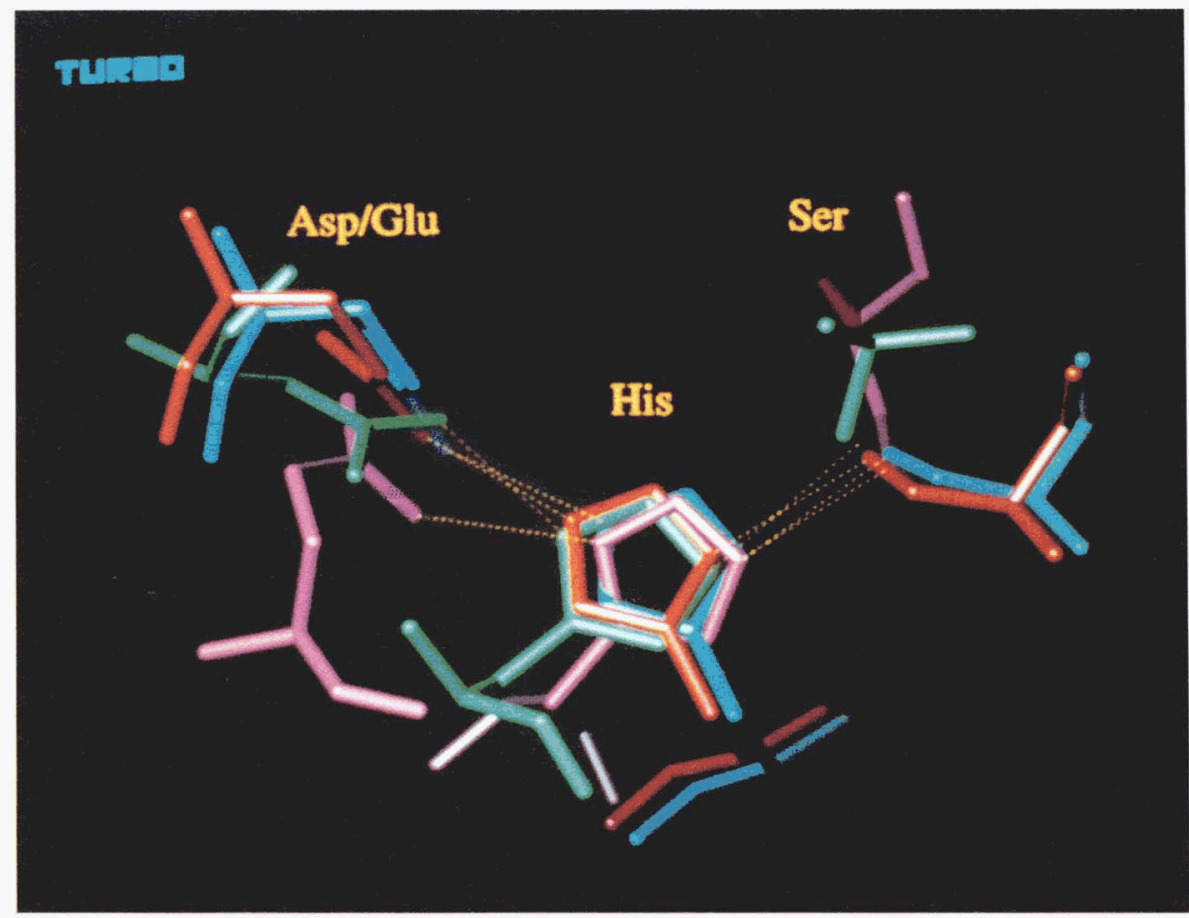

Fig. 7. Same representation as in Figure 6, but here the functional atoms have been superimposed. Note the dramatic rotation of the imidazole ring in A. haloplanctis and human salivary $\alpha$-amylases (in blue and red) compared to those of $G$. candidum lipase (pink) and bovine pancreas $\beta$-trypsin (green).

forming the five binding subsites and having direct or watermediated contacts with the pseudosaccharide inhibitor acarbose in complex with PPA (Qian et al., 1994) are strictly conserved in the psychrophilic $\alpha$-amylase structure. Therefore, no point mutations occur in the catalytic center of $A$. haloplanctis $\alpha$-amylase indicating that a hypothesis of mutations affecting the flexibilty should be preferred, at least in the case of this enzyme. No significant difference is observed in the $B$-factors of these 24 amino acids, giving an average $B$-factor of $18.2 \AA^{2}$ in the case of AHA and $15.4 \AA^{2}$ for the residues from PPA, but one has to be very careful in drawing conclusions as the crystal packing has to be taken into consideration. Concerning the $B$-factors of the whole structure (water molecules not included) of the mesophilic mammalian $\alpha$-amylases, these are not necessarily lower than for the psychrophilic $\alpha$-amylase The average $B$-factors are $13.8 \AA^{2}$ for PPA, $21.5 \AA^{2}$ for HPA, $24.3 \AA^{2}$ for HSA, and $18.9 \AA^{2}$ for AHA. Structural factors susceptible to favour the $\alpha$-amylase conformational flexibility have amongst others been found in the reduced number of salt-bridges, of Arg-mediated hydrogen bonds, of proline residues in loops, and in the low hydrophobicity of the core clusters. A detailed account of these features, based on the comparison of the three-dimensional structure of AHA with its mesophilic and thermophilic (if available) counterparts, will be given in a forthcoming paper.

\section{Materials and methods}

\section{Crystallization, data collection, and processing}

Centered orthorhombic crystals of wild-type AHA complexed with Tris were grown in solutions of MPD by macroseeding as described elsewhere (Aghajari et al., 1996). Native wild-type AHA was found to crystallize under similar conditions. These crystals belong to the space group $\mathrm{C} 222_{1}$, and one molecule is found in the asymmetric unit. Unit cell dimensions for the crystals are given in Table 2. Data were collected on a MARresearch Image plate system, and the X-ray radiation used was $\mathrm{CuK}_{\alpha}$ from a Rigaku RU200 rotating anode operated at $40 \mathrm{kV}$ and $80 \mathrm{~mA}$, with a graphite monochromator. One crystal of free AHA and one crystal of AHA complexed with Tris were used to collect the data sets. Data reductions were performed with DENZO (Otwinowski, 1993; Minor, 1993) and further processing of data were performed with programs from the CCP4 suite (CCP4, 1994).

\section{Structure solution and refinement}

The structure of the AHA/Tris complex was solved by the method of molecular replacement using the native structure of porcine pancreas $\alpha$-amylase (Qian et al., 1993) as a search model and the solution was found with the program AMoRe (Navaza, 1994). Water molecules as well as $\mathrm{Ca}^{2+}$ and $\mathrm{Cl}^{-}$ions were omitted from the search model in order not to bias the search. The molecular replacement search gave a unique solution with a correlation factor of 39.6 and an $R$-factor of $43.2 \%$. Manual fitting and replacement of amino acid residues were carried out using TURBO-FRODO (Roussel \& Cambillau, 1991) and the structures were subsequently refined using programs from the X-PLOR package (Brünger et al., 1987; Brünger et al., 1990) where the Engh and Huber force-field parameters (Engh \& Huber, 1991) were used throughout the course of refinement. Calcium and chloride ions were identified on the basis of coordination, distance, and sigma level criteria. The sigma levels for the calcium and chloride ions were very significative, being 25 and 19 , respectively.

The native AHA structure was solved using phases from the AHA/Tris complex where the Tris molecule as well as water molecules around the active site had been removed 
In both structures, the side chain of Glu 118 had no corresponding electron density and was therefore refined as an alanine. In all refinements performed, data in the range 43-2.0/1.85 $\AA$ (native/ complex data) were used, and the bulk solvent correction (Jiang \& Brünger, 1994) included in the slow-cooling protocol was applied. Data refinement statistics are presented in Table 2. The free $R$-factors (Brünger, 1992) were calculated on the basis of $10 \%$ of the data which have been randomly selected. Coordinates for the two structures have been deposited in the Protein Data Bank with entry codes $1 \mathrm{AQH}$ and $1 \mathrm{AQM}$ for native $\mathrm{AHA}$ and $\mathrm{AHA}$ in complex with Tris, respectively, and will be released with a delay of 1 year.

\section{Acknowledgments}

This work and N.A. were supported by the Human Capital and Mobility Network CHRX-CT94-0521. Support from the Centre National de la Recherche Scientifique is also gratefully acknowledged.

\section{References}

Aghajari N, Feller G, Gerday C, Haser R. 1996. Crystallization and preliminary $\mathrm{X}$-ray diffraction studies of $\alpha$-amylase from the antarctic psychrophile $A l$ teromonas haloplanctis A23. Protein Sci 5:2128-2129.

Blow D. 1990. More of the catalytic triad. Nature 343:694-695.

Boel E, Brady L, Brzozowski AM, Derewenda Z, Dodson GG, Jensen VJ, Petersen SB, Thim L, Woldike HF. 1990. Calcium binding in $\alpha$-amylases: An X-ray diffraction study at $2.1 \AA$ resolution of two enzymes from Aspergillus. Biochemistry 29:6244-6249.

Brady RL, Brzozowski AM, Derewenda ZS, Dodson EJ, Dodson GG. 1991. Solution of the structure of Aspergillus niger acid $\alpha$-amylase by combined molecular replacement and multiple isomorphous replacement methods. Acta Crystallogr B47:527-535.

Brayer GD, Luo Y, Withers SG. 1995. The structure of human pancreatic $\alpha$-amylase at $1.8 \AA$ resolution and comparison with related enzymes. Protein Sci 4:1730-1742.

Brünger AT. 1992. The free R-value: A novel statistical quantity for assessing the accuracy of crystal structures. Nature 355:472-474.

Brünger AT, Krukowski A, Erickson J. 1990 Slow-cooling protocols for crystallographic refinement by simulated annealing. Acta Crystallogr A46:585593.

Bringer AT, Kuriyan M, Karplus M. 1987. Crystallographic R factor refinement by molecular dynamics. Science 235:458-460.

Buisson G, Duée E, Haser R, Payan F. 1987. Three-dimensional structure of porcine pancreatic $\alpha$-amylase at $2.9 \AA$ resolution. Role of calcium ion in structure and activity. EMBO J 6:3909-3916.

Chen C-C, Guo W-J, Isselbacher KJ. 1987. Rat intestinal trehalase. Studies of the active site. Biochem $J$ 247:715-724.

Collaborative Computing Project Number 4. 1994. The CCP4 suite: Programs for protein crystallography. Acta Crystallogr D50:760-763.

Davies G, Henrissat B. 1995. Structures and mechanisms of glycosyl hydrolases. Structure 3:853-859

Engh RA, Huber R. 1991. Accurate bond and angle parameters for X-ray protein structure refinement. Acta Crystallogr A47:392-400.

Feller G, Arpigny JL, Narinx E, Gerday C. 1997. Molecular adaptations of enzymes from psychrophilic organisms. Comp Biochem Physiol 118A:495499.

Feller G, le Bussy O, Houssier C, Gerday C. 1996a. Structural and functional aspects of chloride binding to Alteromonas haloplanctis $\alpha$-amylase. $J$ Biol Chem 271:23836-23841.

Feller G, Lonhienne T, Deroanne C, Van Beeumen J, Gerday C. 1992. Purification, characterization and nucleotide sequence of the thermolabile $\alpha$-amylase from the Antarctic psychrotroph Alteromonas haloplanctis A23.J Biol Chem 267:5217-5221.

Felter G, Narinx E, Arpigny JL, Aittaleb M, Baise E, Gerday C. 1996b. Enzymes from psychrophilic organisms. FEMS Microbiol Rev 18:189-202

Feller G, Payan F, Theys F, Qian M, Haser R, Gerday C. 1994. Stability and structure analysis of $\alpha$-amylase from the antaretic psychrophile Alteromonas haloplanctis A23. Eur J Biochem 222:441 447.

Ishikawa K, Matsui I, Honda K, Nakatani H. 1990. Substrate-dependent shift of optimum $\mathrm{pH}$ in porcine pancreatic $\alpha$-amylase-catalyzed reactions. Biochemistry 29:7119-7123.

Janecek S. 1994. Sequence similarities and evolutionary telationships of microbial, plant and animal $\alpha$-amylases. Eur J Biochem 224:519-524

Jiang J-S, Brünger AT. 1994. Protein hydration observed by X-ray diffraction
Solvation properties of penicillopepsion and neuraminidase crystal structures. I Mol Biol 243:100-115.

Jørgensen BB, Jørgensen OB. 1967. Inhibition of barley malt $\alpha$-glucosidase by Tris(hydroxymethyl)aminomethane and erythritol. Biochim Biophys Acta 146:167-172.

Kadziola A. 1993. An $\alpha$-amylase from barley and its complex with a substrate analogue [Ph.D. thesis]. Marseille, France: CNRS-Marseille and University of Copenhagen.

Kadziola A, Abe J-I, Svensson B, Haser R. 1994. Crystal and molecular structure of barley $\alpha$-amylase. $J$ Mol Biol 239:104-121.

Kadziola A, Sogaard M, Svensson B, Haser R. 1998. Molecular structure of a barley $\alpha$-amylase-inhibitor complex: Inplications for starch binding and catalysis. I Mol Biol. Forthcoming.

Kersters-Hilderson H, Loontiens FG, Claeyssens M, De Bruyne CK. 1969. Partial purification and properties of an induced $\beta$-D-xylosidase of Bacillus pumilus 12. Eur $J$ Biochem 7:434-441.

Koshland DE. 1953. Stereochemistry and the mechanism of enzymatic reactions. Biol Rev 28:416-436.

Koszelak S, Ng JD, Day J, Ko TP, Greenwood A, McPherson A. 1997. The crystallographic structure of the subtilisin protease from Penicillium cyclopium. Biochemistry 36:6597-6604.

Larson SB, Greenwood A, Cascio D, Day J, McPherson A. 1994. Refined molecular structure of pig pancreatic $\alpha$-amylase at $2.1 \AA$ resolution. $J \mathrm{Mol}$ Biol 235:1560-1584.

Levitzki A, Steer ML. 1974. The allosteric activation of mammalian $\alpha$-amylase by chloride. Eur $J$ Biochem $41: 171-180$

Machius M, Wiegand G, Huber R. 1995. Crystal structure of calcium-depleted Bacillus licheniformis $\alpha$-amylase at $2.2 \AA$ resolution. $J$ Mol Biol 246:545559.

Matsuura Y, Kunusoki M, Harada W, Kakudo M. 1984. Structure and possible catalytic residues of Taka-amylase A. J Biochem 95:697-702.

McCarter JD. Withers SG. 1996. Unequivocal identification of Asp-214 as the catalytic nucleophile of Saccharomyces cerevisiae $\alpha$-glucosidase using 5-fluoro glycosyl fluorides. J Biol Chem 271:6889-6894.

Minor W. 1993. XDISPLAYF program. West Lafayette, Indiana: Purdue University Press.

Myrback K. 1965. Studies on yeast $\beta$-fructofuranosidase (invertase) XVII.Inhibition by 2-amino-2-hydroxymethylpropan-1,3-diol. Arkiv fur kemi. 30:315320 .

Navaza J. 1994. AMoRe: An automated package for molecular replacement Acta Crystallogr A50:157-163

Otwinowski Z. 1993. Oscillation data reduction program. In: Sawyer L. Isaacs N, Bailey S, eds. Proceedings of the CCP4 study weekend: Data collection and processing. England: SERC Daresbury Laboratory. pp 56-62.

Qian M, Haser R, Buisson E, Duee E, Payan F. 1994. The active center of a mammalian $\alpha$-amylase. Structure of the complex of a pancreatic $\alpha$-amylase with a carbohydrate inhibitor refined to $2.2-\AA$ resolution. Biochemistry 33:6284-6294.

Qian M, Haser R, Payan F. 1993. Structure and molecular model refinement of pig pancreatic $\alpha$-amylase at 2.1 $\AA$ resolution. I Mol Biol 231:785799

Ramasubbu N, Paloth V, Luo Y, Brayer GD, Levine MJ. 1996. Structure of human salivary $\alpha$-amylase at $1.6 \AA$ resolution: Implications for its role in the oral cavity. Acta Crystallogr D52:435-446.

Roussel A, Cambillau C. 1991. Siticon graphics geometry partners directory 86 Mountain View, CA: Silicon Graphics.

Schrag JD, Li Y, Wu S, Cygler M. 1991. Ser-His-Glu triad forms the catalytic site of the lipase from Geotrichum candidum. Nature 351:761-764.

Søgaard M, Kadziola A, Haser R, Svensson B. 1993. Site-directed mutagenesis of histidine 93, aspartic acid 180, glutamic acid 205, histidine 290, and aspartic acid 291 at the active site and tryptophan 279 at the raw starch binding site in barley $\alpha$-amylase 1. J Biol Chem 268:22480-22484

Strokopytov B, Penninga D, Rozeboom HI, Kalk KH, Dijkhuizen L, Dijkstra BW. 1995. X-ray structure of cyclodextrin glycosyl transferase complexed with acarbose. Implications for the catalytic mechanism of glycosidases. Biochemistry 34:2234-2240.

Svensson B, Søgaard M. 1993. Mutational analysis of glycosilase function. $f$ Biotechnol 29:1-37.

Swift HJ, Brady L, Derewenda ZS, Dodson EJ, Dodson GG, Turkenberg JP, Wilkinson AJ. 1991. Structure and molecular model refinement of Aspergillus oryzae (TAKA) $\alpha$-amylase: An application of the simulated-annealing method. Acta Crystallogr B47:535-544.

Thoma JA, Rao GVK, Brothers C, Spradlin J. 1971. Subsite mapping of enzymes. I Biol Chem 246:5621-5635.

Vihinen M, Mäntsälä P. 1989. Microbial amylolytic enzymes. Crit Rev Biochem Mol Biol 24:329-418.

Vyas NK. 1991. Atomic features of protein-carbohydrate interactions. Cur Opin Struct Biol 1:723-740. 